\title{
A parsimonious approach for screening moderate-to-profound hearing loss in a community-dwelling geriatric population based on a decision tree analysis
}

Min Zhang ${ }^{1 \dagger}$, Zhaori $\mathrm{Bi}^{2+}$, Xinping Fư ${ }^{3}$, Jiaofeng Wang ${ }^{1}$, Qingwei Ruan ${ }^{1}$, Chao Zhao ${ }^{2}$, Jirong Duan ${ }^{3}$, Xuan Zeng ${ }^{4}$, Dian Zhou ${ }^{5}$, Jie Chen ${ }^{1}$ and Zhijun Bao ${ }^{2,1^{*}}$ (D)

\begin{abstract}
Background: Hearing loss is one of the most common modifiable factors associated with cognitive and functional decline in geriatric populations. An accurate, easy-to-apply, and inexpensive hearing screening method is needed to detect hearing loss in community-dwelling elderly people, intervene early and reduce the negative consequences and burden of untreated hearing loss on individuals, families and society. However, available hearing screening tools do not adequately meet the need for large-scale geriatric hearing detection due to several barriers, including time, personnel training and equipment costs. This study aimed to propose an efficient method that could potentially satisfy this need.

Methods: In total, 1793 participants ( $\geq 60$ years) were recruited to undertake a standard audiometric air conduction pure tone test at 4 frequencies $(0.5-4 \mathrm{kHz})$. Audiometric data from one community were used to train the decision tree model and generate a pure tone screening rule to classify people with or without moderate or more serious hearing impairment. Audiometric data from another community were used to validate the tree model.

Results: In the decision tree analysis, $2 \mathrm{kHz}$ and $0.5 \mathrm{kHz}$ were found to be the most important frequencies for hearing severity classification. The tree model suggested a simple two-step screening procedure in which a $42 \mathrm{~dB}$ $\mathrm{HL}$ tone at $2 \mathrm{kHz}$ is presented first, followed by a $47 \mathrm{~dB} \mathrm{HL}$ tone at $0.5 \mathrm{kHz}$, depending on the individual's response to the first tone. This approach achieved an accuracy of $91.20 \%(91.92 \%)$, a sensitivity of $95.35 \%(93.50 \%)$ and a specificity of $86.85 \%(90.56 \%)$ in the training dataset (testing dataset).

Conclusions: A simple two-step screening procedure using the two tones ( $2 \mathrm{kHz}$ and $0.5 \mathrm{kHz}$ ) selected by the decision tree analysis can be applied to screen moderate-to-profound hearing loss in a community-based geriatric population in Shanghai. The decision tree analysis is useful in determining the optimal hearing screening criteria for local elderly populations. Implanting the pair of tones into a well-calibrated sound generator may create a simple, practical and time-efficient screening tool with high accuracy that is readily available at healthcare centers of all levels, thereby facilitating the initiation of extensive nationwide hearing screening in older adults.
\end{abstract}

Keywords: Community-dwelling geriatrics, Hearing screening, Decision tree

\footnotetext{
* Correspondence: zhijunbao@fudan.edu.cn

${ }^{+}$Min Zhang and Zhaori Bi contributed equally on the article

${ }^{2}$ National Clinical Research Center for Aging and Medicine, Huashan Hospital,

Fudan University, Shanghai, China

'Shanghai Key Laboratory of Clinical Geriatric Medicine, Huadong Hospital,

Fudan University, 221 West Yan'an Road, Shanghai, China

Full list of author information is available at the end of the article
}

(c) The Author(s). 2019 Open Access This article is distributed under the terms of the Creative Commons Attribution 4.0 International License (http://creativecommons.org/licenses/by/4.0/), which permits unrestricted use, distribution, and reproduction in any medium, provided you give appropriate credit to the original author(s) and the source, provide a link to the Creative Commons license, and indicate if changes were made. The Creative Commons Public Domain Dedication waiver (http://creativecommons.org/publicdomain/zero/1.0/) applies to the data made available in this article, unless otherwise stated. 


\section{Background}

Age-related hearing loss adds a significant burden to individuals and society. Compared to their normal hearing peers, individuals with hearing loss are at significantly greater risk of incident dementia [1-3], falls [4, 5], depression [6,7], social isolation [8], loss of independence [9], early retirement and unemployment [10], and hospitalization [11]. Hearing loss ranked as the 13th highest contributor to the global burden of disease in 2002 and is projected to be the 9th leading contributor worldwide in 2030 [12]. Excess medical costs resulting from hearing impairment ranged from $\$ 3.3$ to $\$ 12.8$ billion in the United States $[13,14]$ and were $\$ 11.75$ billion in Australia [15]. However, the adverse consequences of untreated hearing loss are still largely underestimated by society, by senior citizens and by health care professionals [16].

The existing literature supports the hypothesis that treating hearing loss is effective in reducing the aforementioned adverse consequences. Nonetheless, moving towards early treatment requires the early identification of individuals with hearing loss. Hearing impairment is highly prevalent in older adults, affecting 33\% of persons over the age of 50 years, $45 \%$ of persons over the age of 60 years [17] and $63.1 \%$ of the population aged 70 years or older [3]. Despite this, fewer than one-fifth of adults with hearing loss seek or obtain any form of treatment [18]. Age-related hearing loss is severely underrecognized and undertreated.

The gold standard for estimating hearing impairment is clinical pure tone audiometry administered by trained audiologists [19]; however, this method is not feasible for large-scale, population-based epidemiological screening projects because it requires access to high-cost audiological equipment and trained personnel. The availability of an effective and sustainable hearing loss screening strategy that is fast, accurate, and easy to use is crucial and a prerequisite for the implementation of effective intervention programs, especially in developing countries with high population density and few hearing care resources.

A number of time-efficient hearing screening strategies have been proposed over the past few years. Portable screeners, such as Audioscope [20], screen hearing by delivering 4 tones of different frequencies $(0.5,1,2$, and $4 \mathrm{kHz}$ ) at approximately $40 \mathrm{~dB}$. Although this approach provides $90 \%$ sensitivity and requires minimal training to administer, it lacks cost-effectiveness because the equipment is not affordable for primary care clinics at the community level. The hearing handicap inventory for the elderly screening (HHIE-S) questionnaire [21, 22] offers an economic option for hearing screening and can be completed within a few minutes with reasonable sensitivity $(74.6 \% \sim 84.5 \%)[19,23]$. However, self-reporting of hearing loss is strongly impacted by individuals' denial or non-acceptance of hearing loss and, as a result, consistently underestimates the actual prevalence of disability [23]. A growing number of application-based hearing tests have provided an accessible and free hearing screening approach by simulating the standard audiometric testing procedure via the internet and personal smart devices [24]. However, the participation rate is low, as these application programs require initiation by older adults, whose motivation to use new technology is low. In addition, the accuracy of these application-based tests is mixed in the literature [25].

Despite the intensive effort to promote hearing screening in community-based geriatric populations, none of the currently available screening instruments have been systematically implemented, and their correlation to clinical audiometric tests varies. To date, no studies have shown that these screening tools resulted in an increased geriatric hearing screening rate or hearing aid use. There was also a global lack of improvement in hearing loss burden as measured by age-standardized disability-adjusted life years (DALYs) over 25 years (1990 2015) [26], implying that the available screening methods have not been accepted by the target facilities, including clinics with heavy flows of patients and densely populated communities. The barriers of cost, time and training still exist, leaving age-related hearing loss largely underdetected.

No parsimonious and feasible screening instrument to date has been developed to fill the above need. The present study was the first attempt to simplify the clinical standard pure tone audiometric assessment by using a machine learning technology on a large pure tone evaluation dataset collected from communities. The goal was to explore an efficient data-driven approach is applicable in the community-based physical check setting for screening moderate-to-profound hearing loss. Specifically, we aimed to implement decision tree algorithms to determine objectively the acoustical screening criteria for hearing classification.

Decision tree is a nonparametric supervised machine learning method used for classification and regression. It creates a practical model that classifies target conditions or predicts the value of a target variable by learning simple decision rules inferred from a set of training data features with a known output. The features most highly related to the outcome are included in the model. Decision tree methodology is ideal for building clinically useful classification models because it uses simple logic for classifying conditions, making it easy for patients and clinicians to interpret data [27-30].

The decision tree model, as a basic machine learning form, is playing an increasingly important role in healthcare applications. Specifically in the hearing science field, 
this technique has been used to seek the optimal suprathreshold test battery to classify auditory profiles towards effective hearing loss compensations [31]. More frequent applications of a decision tree analysis were evaluating whether a medical and audiological practice is cost-effective, for example, implanting cochlear prosthesis [32], the pursuit of magnetic resonance imaging (MRI) with or without contrast in the workup of undifferentiated asymmetrical sensorineural hearing loss [33], and universal or selective hearing screening on newborns [34, 35]. Some sophisticated machine learning technologies (e.g., neural network multilayer perceptron, support vector machine, random forest, adaptive boosting) have also been used in the hearing healthcare area, for instance, predicting postoperative monosyllabic word recognition performance in adult cochlear implant recipients [36] or predicting noise-induced hearing loss of manufacture workers based on demographic information and working acoustical environments [37, 38]. However, to the best of our knowledge, machine learning technologies have not yet been applied in the geriatric hearing screening area for the purpose of a practical implementation.

A decision tree analysis is commonly used to help identify a strategy most likely to reach a goal. In the context of the current study, a decision tree can produce a simple explicative model to determine which pure tone frequencies and intensities could maximize the screening test efficiency. A decision tree has proven a valuable tool for extracting meaningful information from measured data and represents a plausible solution for massive data learning tasks [39]. Additionally it has the advantages of a nonparametric setup, the tolerance of heterogeneous data, and the immunity to noise [40]. The detailed model deduction theory can be found in Breiman and Friedman [41], Friedman [42] and Quinlan [43]. In contrast, most advanced machine learning models answer "yes/no" questions by generating complex structural functions rather than specific values. For example, the neural networks models produce trained functions dependent on network topology and weighted factors [44]. A stochastic based method named Naive Bayes [45, 46] generates a statistical distribution network, which can hardly be parsed as a deterministic function. Although combining multiple decision trees in a random forest [47] may offer better prediction accuracies, given the small number of predictive features and the specific goal of developing explicit classification rules for hearing screening, we concluded that the decision tree method was sufficient to meet our needs.

The research question guiding the present study was whether there is a parsimonious pure tone set that can predict moderate-to-profound hearing loss based on the clinical standard audiometric pure tone thresholds measured in the community-dwelling geriatric population. The classification boundary of moderate hearing loss (pure tone average PTA $>40 \mathrm{~dB} \mathrm{HL}$ ) was selected because moderate or greater hearing loss in older adults is significantly associated with an increased risk of developing frailty [48], lower levels of physical activity [49], and a $20 \%$ increased risk of mortality after adjusting for demographics and cardiovascular risk factors [50]. More importantly, older adults with moderate-to-profound hearing loss benefit from hearing aids or cochlear implants not only in terms of improved hearing function but also in terms of positive effects on anxiety, depression, health status, and quality of life [51]. Our hypothesis was that a pair of tones with specific intensities calculated via decision tree analysis on the pure tone thresholds at four key frequencies (i.e., $500 \mathrm{~Hz}, 1 \mathrm{kHz}, 2$ $\mathrm{kHz}$ and $4 \mathrm{kHz}$ ) could be used to detect moderate-toprofound hearing loss with high $(>85 \%)$ accuracy, sensitivity and specificity.

\section{Methods \\ Participants}

Adults aged 60 years or older from two typical communities (with populations of approximately 110,000 and $84,000)$ in Shanghai were recruited to undertake clinical audiometric pure tone testing. No history of audiological rehabilitation experience (e.g., amplification, auditory training) by self-report was required to participate in the study. A total of 1322 participants were recruited from the Jiuting community (community A), yielding a final sample size of $n=1261$ (mean age $=71.4$ yrs., range 6092) after excluding 61 participants due to missing audiometric data from either ear. Of the 536 participants recruited from the Nanmatou community (community B), 4 were excluded from the analysis due to missing audiometric data, yielding a final sample size of $n=532$ (mean age $=76.5$ yrs., range $60-104$ ). The demographic information regarding age, sex and hearing severity are displayed in Table 1.

\section{Audiometric assessment}

Audiometric pure tone testing was administered by two trained audiologists in a quiet consulting room in each of the community healthcare centers located within a 15-min walking distance from the residents' homes. Prior to the audiometric assessment, the ears were examined for wax or abnormalities. The air conduction thresholds were obtained at $0.5 \sim 4 \mathrm{kHz}$ over an intensity range of -10 to $120 \mathrm{~dB}$ using an Interacoustics MA52 audiometer with manual testing per protocol. Each individual's hearing thresholds were annotated as $\mathrm{dB} H \mathrm{HL}$ at each of 4 frequencies $(500 \mathrm{~Hz}, 1 \mathrm{kHz}, 2 \mathrm{kHz}$ and $4 \mathrm{kHz})$. Stimuli were presented through supra-aural headphones (TDH-39), except in rare cases of ear canal collapse or 
Table 1 Demographic Characteristics of Participants Aged 60 Years or Older With Audiometric Testing. The degree of hearing loss was classified based on the pure tone average thresholds (PTA) of the better and worse ear

\begin{tabular}{|c|c|c|c|c|c|c|c|c|}
\hline & \multicolumn{4}{|c|}{ Community A $(n=1261)$} & \multicolumn{4}{|c|}{ Community B $(n=532)$} \\
\hline & \multicolumn{2}{|c|}{ Better-hearing ear } & \multicolumn{2}{|c|}{ Worse-hearing ear } & \multicolumn{2}{|c|}{ Better-hearing ear } & \multicolumn{2}{|c|}{ Worse-hearing ear } \\
\hline & $\begin{array}{l}\mathrm{NH} \\
(n=18)\end{array}$ & $\begin{array}{l}\mathrm{HL} \\
(n=1243)\end{array}$ & $\begin{array}{l}\mathrm{NH} \\
(n=9)\end{array}$ & $\begin{array}{l}\mathrm{HL} \\
(n=1252)\end{array}$ & $\begin{array}{l}\mathrm{NH} \\
(n=84)\end{array}$ & $\begin{array}{l}\mathrm{HL} \\
(n=448)\end{array}$ & $\begin{array}{l}\mathrm{NH} \\
(n=33)\end{array}$ & $\begin{array}{l}\mathrm{HL} \\
(n=499)\end{array}$ \\
\hline \multicolumn{9}{|l|}{ Sex } \\
\hline Male & $6(33.3 \%)$ & $540(43.4 \%)$ & $5(55.6 \%)$ & $541(43.2 \%)$ & $30(35.7 \%)$ & $294(34.4 \%)$ & $8(24.2 \%)$ & $176(35.3 \%)$ \\
\hline Female & $12(66.7 \%)$ & $703(56.6 \%)$ & $4(44.4 \%)$ & $711(56.8 \%)$ & $54(64.3 \%)$ & $154(65.6 \%)$ & $25(75.8 \%)$ & $323(64.7 \%)$ \\
\hline \multicolumn{9}{|l|}{ Age group } \\
\hline $60-69$ & $8(44.4 \%)$ & $526(42.3 \%)$ & $4(44.4 \%)$ & $53042.0 \%)$ & $51(60.7 \%)$ & $118(26.2 \%)$ & $25(75.8 \%)$ & $144(28.9 \%)$ \\
\hline $70-79$ & $9(50 \%)$ & $609(49 \%)$ & $4(44.4 \%)$ & $614(49.3 \%)$ & $22(26.2 \%)$ & $127(28.3 \%)$ & $6(18.2 \%)$ & $143(28.7 \%)$ \\
\hline$\geq 80$ & $1(5.6 \%)$ & $108(8.8 \%)$ & $1(11.2 \%)$ & $108(8.7 \%)$ & $11(13.1 \%)$ & $203(45.4 \%)$ & $2(6.0 \%)$ & $212(42.4 \%)$ \\
\hline \multicolumn{9}{|l|}{ Hearing symmetry } \\
\hline Symmetrical PTA & $18(100 \%)$ & $1197(96.3 \%)$ & $9(100 \%)$ & $1206(96.3 \%)$ & $79(94 \%)$ & $402(89.7 \%)$ & $33(100 \%)$ & $447(89.6 \%)$ \\
\hline Asymmetrical PTA & 0 & $46(3.7 \%)$ & 0 & $46(3.7 \%)$ & $5(6 \%)$ & $46(10.3 \%)$ & 0 & $52(10.4 \%)$ \\
\hline \multicolumn{9}{|c|}{ Hearing severity based on the WHO standard } \\
\hline Mild (PTA 26 40 dB HL) & & $598(47.4 \%)$ & & $360(28.8 \%)$ & & $202(38 \%)$ & & $169(33.9 \%)$ \\
\hline Moderate (PTA 41 70 dB HL) & & $576(45.7 \%)$ & & $725(57.9 \%)$ & & $176(33.1 \%)$ & & $191(38.3 \%)$ \\
\hline Severe (PTA 71 90 dB HL) & & $61(4.8 \%)$ & & $135(10.8 \%)$ & & $60(11.3 \%)$ & & $90(18.0 \%)$ \\
\hline Profound (PTA > $90 \mathrm{~dB} \mathrm{HL}$ ) & & $8(0.6 \%)$ & & $32(2.5 \%)$ & & $10(1.9 \%)$ & & 49 (9.8\%) \\
\hline
\end{tabular}

crossover retesting when insert earphones (Ear Tone 3A) were used. As acoustic isolation was not available, the ambient noise levels were monitored during the pure tone testing by an AWA5636 sound level meter. The average ambient noise level ranged between 35 and $38 \mathrm{dBA}$.

Hearing loss severity was defined per the classification of the World Health Organization as a pure tone threshold (PTA at 0.5, 1, 2 and $4 \mathrm{kHz}$ ) between $25 \mathrm{~dB} \mathrm{HL}$ and $40 \mathrm{~dB} \mathrm{HL}$ indicating mild hearing impairment and a hearing threshold of more than $40 \mathrm{~dB} \mathrm{HL}$ indicating moderate or more severe hearing impairment. Data were collected over a 4-month period in community $\mathrm{A}$ and a 2.5-month period in community B.

\section{Analysis strategy}

The descriptive statistics related to the community sample characteristics were evaluated using PASW 24 (SPSS/IBM, Chicago, IL). Pearson's chi-square test was employed for the analysis of proportions comparison. Regression analysis was performed to test the relationship between age and hearing acuity. The level of significance was established at the 0.05 level.

The decision tree analysis was performed using the Python 3.7.0 SKlearn 0.19.2 package. We used a decision tree approach with a depth of 2 levels to analyze the audiometric pure tone data. A deeper tree was not considered in this study because the number of features in the model (i.e., 4 frequencies) associated with the hearing severity determination was fairly small, and the goal of the study was to simplify the hearing assessment procedure by using only 2 of the 4 frequencies. The known classification output for model training and testing was two classes of hearing status labeled as "normal-to-mild hearing loss (PTA $<=$ $40 \mathrm{~dB} \mathrm{HL}$ )" and "moderate-to-profound hearing loss (PTA $>40 \mathrm{~dB}$ HL)". Data collected from community A ( $n=1261$, i.e., $70.3 \%$ of the dataset) served as the training dataset, and data collected from community B $(n=532$, i.e., $29.7 \%$ of the total datasets) served as the test dataset in the decision tree analysis.

A brief mathematical illustration of the tree-based machine learning methodology used in the current study is displayed in Fig. 1. We defined the input variable (i.e., determinant pure tone frequency) as $X_{i}$, where $X_{i} \in \Omega_{i}$ corresponds to the variable of the $i$-th input space. The ideal learning function is defined as $f: X \rightarrow Y$, where $Y \in\left\{c_{1}, c_{2}, \ldots, c_{n}\right\}$ is a finite set of labels for the classification problem. A tree-based model is a special representation of $f$ with a rooted tree whose node $t$ partitions the input space into the subspace $\Omega_{t}$, as shown in Fig. 1a. Ultimately, terminal nodes $t_{c i}$ represent the best guess of $\hat{Y} \in\left\{\widehat{c_{1}}, \widehat{c_{2}}, \ldots, \widehat{c_{n}}\right\}$. The selection of the attribute used at each node of the tree to split the data is crucial for correct classification. Different split criteria (functions) were proposed in the literature [52], and we applied two widely used impurity functions in the study: Shannon entropy [53] and the Gini index [54]. Equations (1) and 

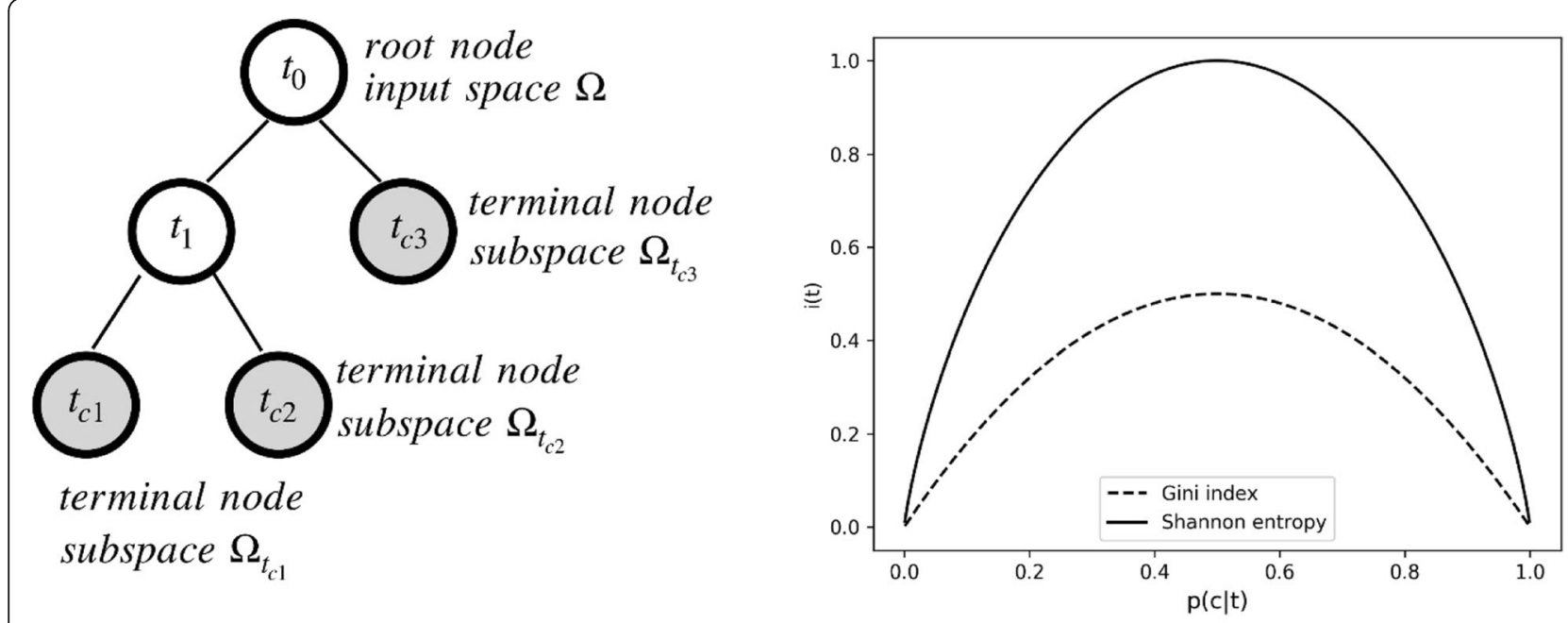

a

b

Fig. 1 a Decision tree graph form, node definitions and space divisions. b Impurity function plotting. The solid line represents the Shannon entropy function, and the dashed line represents the Gini index

(2) quantified the uncertainty function $i(t)$ with node $t$ classification ratio $p\left(c_{k} \mid t\right)=N_{c_{k} t} / N_{t}$, where $N$ is the number of samples.

$$
\begin{aligned}
& i_{\text {Shannon }}(t)=-\sum_{k=1}^{m} p\left(c_{k} \mid t\right) \log _{2}\left(p\left(c_{k} \mid t\right)\right) \\
& i_{\text {Gini }}(t)=\sum_{k=1}^{m} p\left(c_{k} \mid t\right)\left(1-p\left(c_{k} \mid t\right)\right)
\end{aligned}
$$

The above functions were plotted in Fig. 1b.

Conventionally in the clinical hearing severity classification, all 4 frequencies are assumed to be equally important and are required upon a hearing assessment when determining severity. To simplify the procedure for screening purposes, we computed the importance rank of the 4 frequencies through the tree structure training process, seeking to discover the 2 most important frequencies of the 4 for classification with high accuracy. The importance weight of each frequency was computed using Eq. (3) [41]:

$$
\text { Importance }=N_{t} / N *\left(i(t)-N_{t r} / N_{t} * i\left(t_{r}\right)-N_{t l} / N_{t} * i\left(t_{l}\right)\right)
$$

where $N$ is the total number of samples, $N_{t}$ is the number of samples for the current node, $N_{t r}$ is the number of samples for the child node on the right side of the tree branch, $N_{t l}$ is the number of samples for the child node on the left side of the tree branch, and $i$ is the tobe-optimized impurity function for the corresponding nodes.
In addition to the decision tree analysis, 3 state-of-theart machine learning analyses applicable to our datasets were performed for classification outcome comparisons. These machine learning models included Support Vector Machine (SVM), Random Forest (RF), and Multilayer Perceptron (MLP). The same training and testing data used in the decision tree were fed to these models. The SVM model was fitted with the Radial Basis Function kernel [55]. The RF was set up with a depth of 2 for each of 100 estimators [56]. MLP was constructed with 100 hidden layers, and the optimization solver was set as "adam" [57]. The analyses were implemented in Python with the Scikit-learn package [58].

As an individual's hearing status can be labeled either by the better-hearing ear's PTA according to WHO or based on the worse-hearing ear's PTA for screening purposes as suggested by hearing screening researchers [22, 59], the decision tree analysis was performed on the pure tone thresholds of the better-hearing ear and the worse-hearing ear separately.

\section{Results}

\section{Descriptive analysis}

The demographic characteristics of the participants from the two communities are shown in Table 1. The two geriatric samples displayed a number of distinct characteristics. On average, participants from community A $(\mathrm{M}=71.41, \mathrm{SD}=5.32)$ were significantly younger than participants from community $\mathrm{B}(\mathrm{M}=76.55, \mathrm{SD}=10.18)$, $t(1791)=13.962, p<.001$. The sex ratio of participants with hearing loss showed opposite patterns between the two communities; there were significantly more male than female participants with hearing loss in community 
B, $\lambda^{2}(1)=9.303, p=.002$. The hearing severity distribution was significantly different between the two community samples depending on how hearing loss was defined, $\lambda^{2}(4)=186.086, p<.01$. Specifically, when hearing loss was defined by the better-hearing ear's PTAs, there was no significant difference in the proportion of older people with moderate-to-profound hearing loss between community A (51.9\%) and community B (54.9\%\%), $\lambda^{2}(1)=1.205, p=.272$. However, if hearing severity was categorized according to the worse-hearing ear's PTAs, the prevalence of moderate-to-profound hearing loss was significantly higher in community A (70.7\%) than in community B $(62.0 \%), \lambda^{2}(1)=13.070, p<.01$.

Asymmetrical PTA was defined in the present study as a difference in the four-frequency pure tone average between the worse- and better-hearing ears larger than 20 $\mathrm{dB}$ HL. The PTA difference between better- and worsehearing ears ranged from $0 \mathrm{~dB}$ HL to $55 \mathrm{~dB} \mathrm{HL}$ in the community A sample and from $0 \mathrm{~dB}$ HL to $68.75 \mathrm{~dB} \mathrm{HL}$ in the community B sample. The community A geriatric sample $(3.7 \%)$ had a significantly smaller proportion of asymmetrical PTA than the community B sample (10.4\%), $\lambda^{2}(1)=30.738, p<.01$.

The regression analysis on the PTA predicted by age illustrated that age was significantly associated with hearing acuity $(B=.601, F(1,1791)=256.418, p<.001$, adjusted $\left.R^{2}=.125\right)$. Specifically, the hearing acuity dropped $0.6 \mathrm{~dB}$ for every one-year increase in age above 60 years old.

\section{Decision tree analysis}

The implemented decision tree analysis using two impurity functions generated the same results, namely, that the $0.5 \mathrm{kHz}$ tone and $2 \mathrm{kHz}$ tone were the two most important frequencies for classifying older adults with and without moderate-to-profound hearing loss (Fig. 2).
The tree model suggested a simple two-step screening approach (Fig. 3): the $2 \mathrm{kHz}$ tone with a specific intensity is presented at the first step, followed by a $0.5 \mathrm{kHz}$ tone depending on the individual's response to the $2 \mathrm{kHz}$ tone. The screening tone intensities were determined based on the optimal Gini index and Shannon entropy. When an individual's hearing severity was defined by the better-hearing ear, a combination of a $2 \mathrm{kHz}(42 \mathrm{~dB} \mathrm{HL})$ tone and a $0.5 \mathrm{kHz}(47 \mathrm{~dB} \mathrm{HL})$ tone was suggested. When the worse-hearing ear was selected to determine an individual's hearing status, a combination of a $2 \mathrm{kHz}$ $(37 \mathrm{~dB} \mathrm{HL})$ tone and a $0.5 \mathrm{kHz}(47 \mathrm{~dB} \mathrm{HL})$ tone was suggested.

The performance of the decision tree analysis for moderate-to-profound hearing loss prediction was evaluated in terms of sensitivity, specificity and accuracy calculated from the confusion matrices summarized in Table 2 . The confusion matrices present the detailed predictions produced from the datasets at the optimal threshold based on the Gini index (the Shannon entropy produced the same results). The sensitivity, specificity and accuracy are displayed in Table 3. The decision tree for the better-hearing ear screening achieved a classification accuracy of $91.20 \%$ with a sensitivity of $96.35 \%$ and a specificity of $86.85 \%$ based on the training dataset and obtained a consistent classification outcome when tested with the community B dataset. The decision tree for the worse-hearing ear screening achieved a classification accuracy of $89.93 \%$ with a sensitivity of $97.53 \%$ and a specificity of $71.54 \%$ for the training dataset and obtained a comparable classification outcome when tested with the community B dataset. The participants who were misclassified as having moderate-to-profound hearing loss (false positives) all had mild hearing loss.

The classification performance of the advanced machine learning methods were displayed alongside with

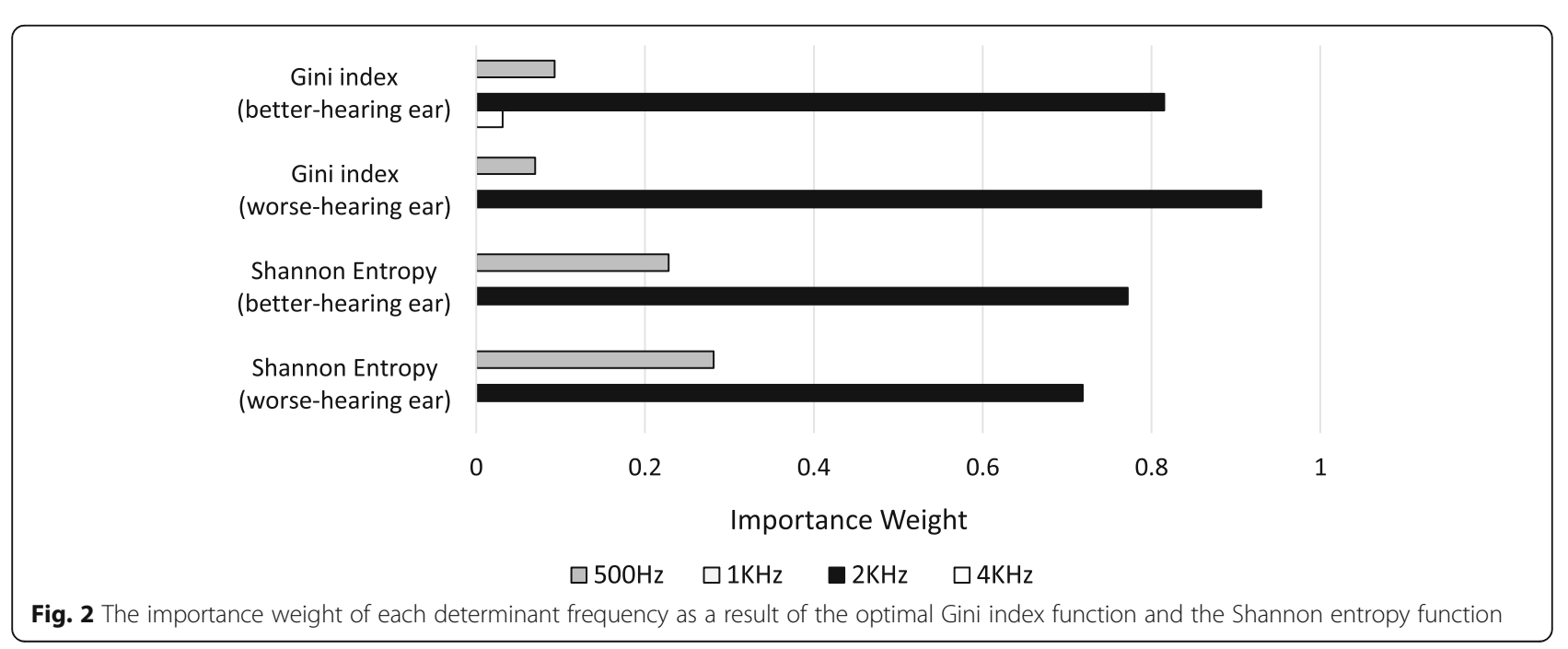




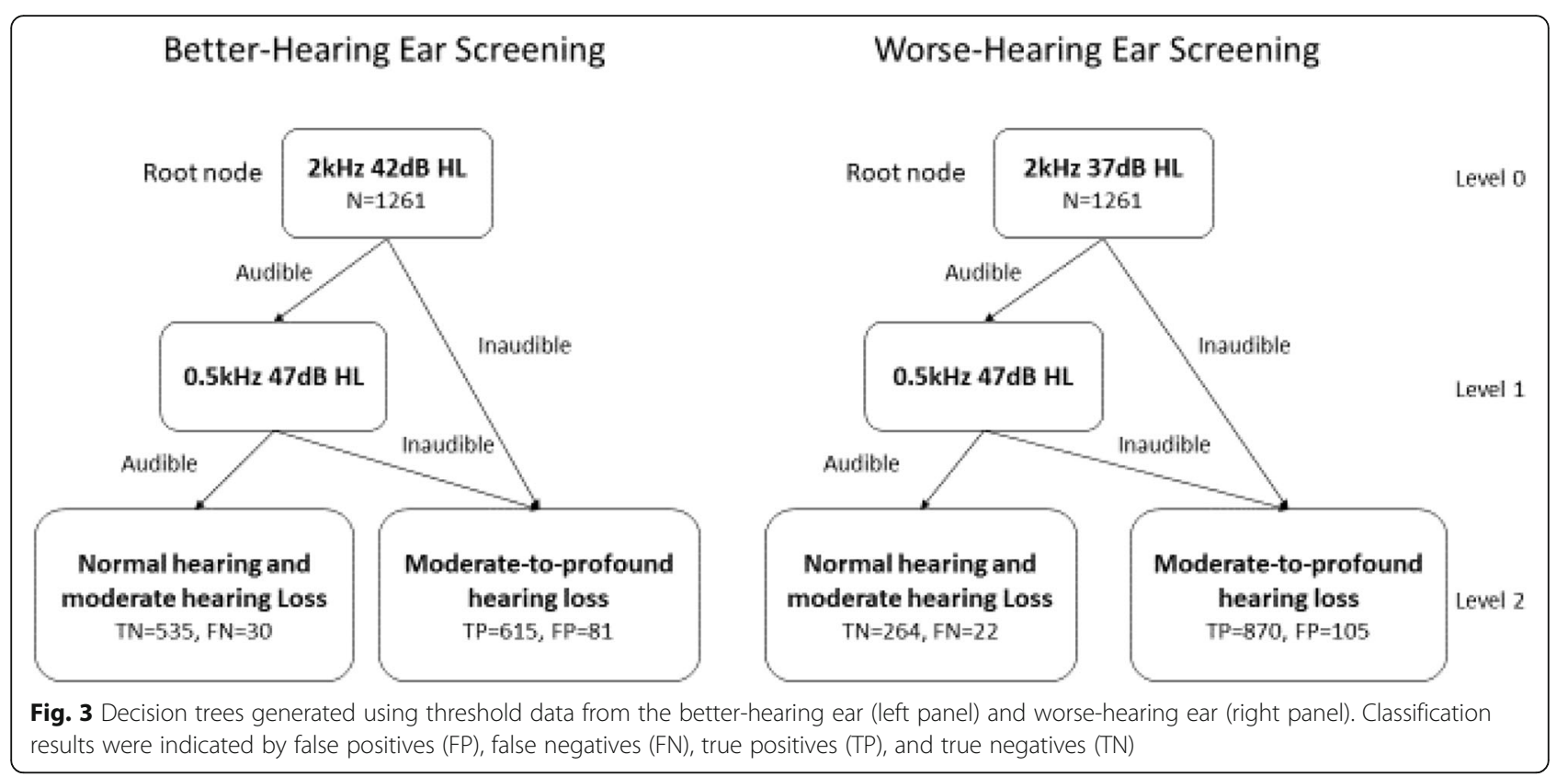

the decision tree outcomes in Table 4. The results suggested that the outcomes of the decision tree approach were comparable with the random forest. Both the decision tree and the random forest were superior to the support vector machine and multilayer perceptron in the current study when comprehensively considering the sensitivity, specificity and accuracy in the test set.

In addition, the intuitive screening criteria of $40 \mathrm{~dB}$ $\mathrm{HL}$ at $2 \mathrm{kHz}$ and $1 \mathrm{kHz}$ suggested by previous literature were tested in our sample as a comparison. The confusion matrix for classifying moderate-to-profound hearing loss in community A indicated a TP of 556, TN of 584, FP of 32 and FN of 89, resulting in an accuracy of $90.4 \%$ (with a sensitivity of $86.20 \%$ and a specificity of $94.81 \%$ ). The classification using $40 \mathrm{~dB}$ HL cutoffs in community B produced an accuracy of $91.35 \%$ (with a sensitivity of $90.00 \%$ and a specificity of $92.55 \%$ ). The performance of different screening criteria is illustrated in Fig. 4.

\section{Discussion}

The current study used a fundamental machine learning technique to explore a parsimonious pair of pure tones that can be applied for hearing screening with a sensitivity, specificity and accuracy over $85 \%$ in communitydwelling older adults over 60 years of age. This was the first effort to objectively simplify the standard clinical pure tone hearing test procedure and construct an acoustic hearing screening criteria feasible for large-scale geriatric hearing screening. The screening tones were determined by collecting standard audiometric testing results from 1793 older community residents and by implementing decision tree analysis on their pure tone thresholds.

In line with Ciurlia-Guy et al.'s [60] finding, our decision tree analysis identified $2 \mathrm{kHz}$ as the most important frequency for moderate-to-profound hearing loss screening in elderly people (Fig. 2). However, distinct from the conventional belief in the important role of $1 \mathrm{kHz}$ in hearing screening, our results indicated that $1 \mathrm{kHz}$ had a minimal impact on hearing classification at the moderate severity level; instead, $0.5 \mathrm{kHz}$ became the second most important frequency. The tree model suggested using a $2 \mathrm{kHz}$ tone coupled with a $0.5 \mathrm{kHz}$ tone to achieve the optimal classification performance. The most appropriate intensities of the screening tones quantified based on the Gini index for the better-hearing ear were $42 \mathrm{~dB} \mathrm{HL}$ at $2 \mathrm{kHz}$ and $47 \mathrm{~dB} \mathrm{HL}$ at $0.5 \mathrm{kHz}$. This procedure increased the sensitivity and accuracy by 9.1 and $1.0 \%$, respectively, compared to using the intuitive $40 \mathrm{~dB} \mathrm{HL}$ at $2 \mathrm{kHz}$ and $1 \mathrm{kHz}$ as the criteria (Fig. 4). Sensitivity is

Table 2 Confusion matrix summarizing the number of false positives (FP), false negatives (FN), true positives (TP), and true negatives (TN). Numbers in parentheses are the count from the test dataset (community B)

\begin{tabular}{lllllll}
\hline & & \multicolumn{2}{l}{ Actual (better-hearing ear) } & & & \multicolumn{2}{l}{ Actual (worse- hearing ear) } \\
\cline { 3 - 4 } & & Positive & Negative & & Positive & Negative \\
\hline Predicted & True & $615(230)$ & $535(259)$ & $870(322)$ & 264 (163) \\
& False & $30(27)$ & $81(16)$ & 22 (8) & 105 (39) \\
\hline
\end{tabular}


Table 3 Sensitivity, specificity and accuracy of classifying moderate-to-profound hearing loss by the computed screening tones

\begin{tabular}{llllll}
\hline & \multicolumn{2}{l}{ Better-hearing ear } & & \multicolumn{2}{l}{ Worse-hearing ear } \\
\cline { 2 - 3 } \cline { 5 - 6 } & Training Set (Community A) & Test Set (Community B) & & Training Set (Community A) & Test Set (Community B) \\
\hline Sensitivity & $95.35 \%$ & $93.50 \%$ & $97.53 \%$ & $97.58 \%$ \\
Specificity & $86.85 \%$ & $90.56 \%$ & $71.54 \%$ & $80.69 \%$ \\
Accuracy & $91.20 \%$ & $91.92 \%$ & $89.93 \%$ & $91.17 \%$ \\
\hline
\end{tabular}

important in the context of this study because early intervention for as many individuals with true moderate or greater hearing loss as possible is desired. On the other hand, specificity also needs to be accounted for to minimize the unnecessary number of false referrals. The improvement of sensitivity by using $42 \mathrm{~dB} \mathrm{HL}$ at $2 \mathrm{kHz}$ is clinically significant when the screening target is a large geriatric population.

The optimal screening criteria computed based on the worse-hearing ear were slightly different at $2 \mathrm{kHz}$ due to the proportion of participants with asymmetrical hearing loss. The cutoff value of $37 \mathrm{~dB}$ HL produced a sensitivity of $97.53 \%$ with an accuracy of $89.93 \%$, which implies a more aggressive screening approach. However, the specificity was compromised by $15.31 \%$, which indicates a high false positive rate that will result in a waste of health resources. The difference in the criteria between the two ears suggests a consideration of what rationale to follow prior to massive screening. If healthcare providers believe that older adults' hearing severity should be defined by the worse ear's PTA and that unilateral hearing loss should be treated in this population, then the criteria computed based on the worse-hearing ear thresholds are recommended. If healthcare providers support that the better ear represents an individual's overall hearing function and an early intervention should be implemented when the better ear has moderate or greater hearing loss, then the criteria calculated based on the better-hearing ear thresholds are recommended.

The results of the current study suggested that the decision tree models constitute useful analytical tools to screen moderate-to-profound hearing loss. The decision tree technique formed a simple explicative model and produced easy-to-interpret results (Fig. 3). Although the random forest produced slightly higher classification sensitivity, specificity and accuracy in the current study, it did not provide an explicit cutoff criteria for practical use. Other advanced machine learning techniques, such as support vector, were able to "learn" considerably well from the training set; however, the trained models did not effectively generalize to new datasets (Table 4), which was reflected by the poor specificities $(<55 \%)$ compromising the overall accuracy $(<80 \%)$. Those stateof-the-art methods did not deduce a cutoff criteria to meet the purpose of the current study either. Compared to those advanced black-box-based machine learning

Table 4 Comparisons on the sensitivity, specificity and accuracy of classification among different machine learning approaches. Performances greater than $85 \%$ are highlighted in bold

\begin{tabular}{|c|c|c|c|c|c|}
\hline & & & Sensitivity & Specificity & Accuracy \\
\hline \multirow[t]{8}{*}{ Better-hearing ear } & \multirow[t]{4}{*}{ Training set (Community A) } & Decision Tree (DT) & $95.35 \%$ & $86.85 \%$ & $91.20 \%$ \\
\hline & & Support Vector Machine (SVM) & $100.00 \%$ & $100.00 \%$ & $100.00 \%$ \\
\hline & & Random Forest (RF) & $91.32 \%$ & $94.97 \%$ & $93.10 \%$ \\
\hline & & Multilayer Perceptron (MLP) & $78.29 \%$ & $56.98 \%$ & $67.88 \%$ \\
\hline & \multirow[t]{4}{*}{ Test set (Community B) } & Decision Tree (DT) & $93.50 \%$ & $90.56 \%$ & $91.92 \%$ \\
\hline & & Support Vector Machine (SVM) & $100.00 \%$ & $33.57 \%$ & $64.29 \%$ \\
\hline & & Random Forest (RF) & $94.31 \%$ & $92.31 \%$ & $93.23 \%$ \\
\hline & & Multilayer Perceptron (MLP) & $90.65 \%$ & $51.05 \%$ & $69.36 \%$ \\
\hline \multirow[t]{8}{*}{ Worse-hearing ear } & \multirow[t]{4}{*}{ Training set (Community A) } & Decision Tree (DT) & $97.53 \%$ & $71.54 \%$ & $89.93 \%$ \\
\hline & & Support Vector Machine (SVM) & $100.00 \%$ & $100.00 \%$ & $100.00 \%$ \\
\hline & & Random Forest (RF) & $98.21 \%$ & $77.51 \%$ & $92.15 \%$ \\
\hline & & Multilayer Perceptron (MLP) & $99.89 \%$ & $11.38 \%$ & $73.99 \%$ \\
\hline & \multirow[t]{4}{*}{ Test set (Community B) } & Decision Tree (DT) & $97.58 \%$ & $80.69 \%$ & $91.17 \%$ \\
\hline & & Support Vector Machine (SVM) & $100.00 \%$ & $34.16 \%$ & $75.00 \%$ \\
\hline & & Random Forest (RF) & $99.39 \%$ & $84.65 \%$ & $93.80 \%$ \\
\hline & & Multilayer Perceptron (MLP) & $100.00 \%$ & $19.31 \%$ & $69.36 \%$ \\
\hline
\end{tabular}




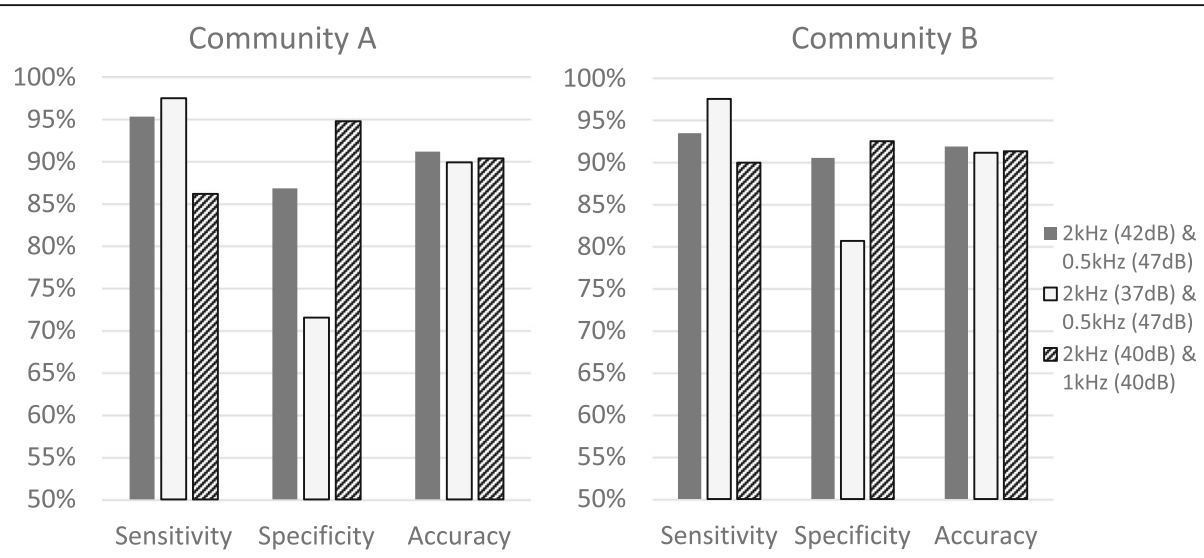

Fig. 4 The sensitivity, specificity and accuracy as a result of different screening approaches. The left panel displays the classification performance using community A's data, and the right panel displays the classification performance using community B's data

algorithms, the decision tree method demonstrated the advantage of knowledge extraction that requires explicit explanations of the data relationships.

The results can be readily translated into feasible clinical applications. In the simplest form, an inexpensive well-calibrated sound generator can be used to incorporate the computed pure tones and deliver them in the correct order to the target individuals. This method integrates the advantages of previous screening approaches. First, its accuracy is as high as that of the screening audiometers reported in the literature (e.g., AudioScope). In both community samples, the accuracy of using the pair of 2 $\mathrm{kHz}$ and $0.5 \mathrm{kHz}$ tones exceeded $91 \%$. Second, since the screening criteria were directly calculated based on the standard pure tone thresholds, the correlation of the screening result with the gold standard audiometric results is inherently strong. Third, screening with acoustically calibrated tones is perceived as more objective than using self-report and uncalibrated methods such as the whisper test [61] by both clinicians and patients and consequently will motivate greater engagement of healthcare providers from multidisciplinary areas in the hearing screening program. Fourth, the simple two-step procedure can save substantial time for clinicians. Screening results can be gathered in less than $1 \mathrm{~min}$ (including delivering the 4 tones ( 2 to each ear) and receiving responses) for each individual. No specialized training is needed, and any healthcare provider is able to determine whether to make a referral for further diagnostic audiometric evaluation and treatment by following the tree model. Finally, this method can be cost-effective. Compared to the currently available screening devices, which are relatively expensive, a sound generator programmed with precise screening tones will be more affordable for health centers at the community level.

The prevalence of moderate-to-profound hearing loss shown in this study's samples $(52$ and $60 \%$ in communities $\mathrm{A}$ and $\mathrm{B}$, respectively) was consistent with the prevalence documented in previous epidemiological studies [23, 62-64] (Table 1). The proportion of older adults with normal hearing was low due to the testing conditions in a quiet but nonsoundproofed room. A portion of the participants with normal hearing were likely to be misdiagnosed as having mild hearing loss, but this had a minimum impact on our results because the classification boundary was set at moderate hearing loss $(\mathrm{PTA}=40$ $\mathrm{dB}$ HL). The ambient noise level in the test environment was under $40 \mathrm{dBA}$, which permitted the accurate evaluation of the moderate-to-profound pure tone thresholds. Asymmetrical hearing loss was not common in our study samples, which is consistent with the literature [65]. The significant positive linear relationship between hearing loss and age found in our study is also in line with previous studies $[17,62]$. Participants in the study were not screened for family history of deafness, dementia or occupational noise exposure, and we believe that they are representative of the general elderly population. Although the age, sex, hearing loss severity and asymmetry distribution characteristics were different between the two community samples, the decision tree model generated by analyzing the data from community A was robust. The tree model resulted in comparable classification performance when tested on the data of community B.

The results of the present study illustrated that hearing loss is largely underrecognized in the communitydwelling geriatric population. Given the increasing prevalence and disease burden of undetected hearing loss in older adults and the availability of effective treatment (e.g., modern amplification devices, aural rehabilitation), early identification of individuals with moderate or greater hearing loss is a cost-saving strategy. However, screening methods at the community level are inadequate. There is an unmet need for a feasible data-driven screening protocol for healthcare policy makers to 
promote early identification and immediate treatment of hearing loss in geriatric patients. The current study demonstrated how a machine learning technique, specifically decision tree analysis, can be used in the hearing healthcare setting to meet the need for a parsimonious acoustical screening protocol for community-dwelling geriatric populations. Community health care centers have the potential to serve as the first point of access to identify moderate or greater hearing loss. The proposed simple two-step pure tone screening approach made it possible for the hearing test to be included in routine checkups. This study suggests a feasible and powerful means to deliver quality services when the gold standard audiometric evaluation of hearing is not available.

The main limitation of our study was that our data were collected from two communities in a single city, which may affect the generalizability of the results. Validating the tree model with audiometric data from communities in a few randomly selected cities and rural areas is needed to generalize the proposed screening instrument nationwide.

\section{Conclusion}

Our study proposed a parsimonious two-step screening procedure using decision tree analysis. A pair of tones $(2$ $\mathrm{kHz}$ and $0.5 \mathrm{kHz}$ ) was identified to screen for moderateto-profound hearing loss in community-based geriatric populations over 60 years of age in Shanghai. Implanting the pair of tones into a well-calibrated sound generator can create a simple, practical and time-efficient screening tool with high accuracy that can be made available at health centers at all levels and can thus facilitate the initiation of nationwide extensive hearing screening in older adults. The decision tree approach is an appropriate method to determine the optimal pure tone hearing screening criteria for local geriatric populations and identify high-risk subpopulations that need early prevention and intervention programs and, therefore, could be used to make the most of public health resources.

\section{Abbreviations}

dB HL: Decibel hearing level; PTA: Pure tone average

\section{Acknowledgements}

We would like to thank all the participants for their participation in the study. We would like to thank the audiologists Xinping Fu and Jiao Wan for collecting the audiometric data in the communities. We would also like to thank the nurse practitioners Wei Chen, Wenjuan Lin, Bei Zhu and Yue Pan for their administrative help, including scheduling appointments and organizing the data files.

\section{Authors' contributions}

All authors listed have met all the International Committee of Medical Journal Editors (ICMJE) criteria for authorship and have contributed equally to this work. ZBi, XZ, DZ and MZ led the statistical analysis. MZ, ZBi and ZBa drafted and redrafted the whole report. FX and JD collected and interpreted the patient data. All authors contributed to sections of the reports and all revised the paper for important intellectual content. All authors read and approved the final manuscript.

\section{Funding}

This research was funded by the National Science and Technology Commission Municipality Research Fund, \#2018YFC2002000.

\section{Availability of data and materials}

The datasets used and analyzed during the current study are available from the corresponding author upon reasonable request.

\section{Ethics approval and consent to participate}

Ethical approval for this study was obtained from Fudan University Huadong Hospital (No. 2018 K153). All participants signed written consent forms. No reimbursement was provided for participation; however, the audiometric assessment results were discussed with those who were identified as having hearing loss. Information about audiology clinic appointments was provided.

\section{Consent for publication}

Not applicable.

\section{Competing interests}

The authors declare that they have no competing interests.

\section{Author details}

'Shanghai Key Laboratory of Clinical Geriatric Medicine, Huadong Hospital, Fudan University, 221 West Yan'an Road, Shanghai, China. ${ }^{2}$ National Clinical Research Center for Aging and Medicine, Huashan Hospital, Fudan University, Shanghai, China. ${ }^{3}$ Speech and Hearing Rehabilitation Department, Punan Hospital, Fudan University, Shanghai, China. ${ }^{4}$ The State Key Laboratory of ASIC \& System, Department of Microelectronics, Fudan University, Shanghai, China. ${ }^{5}$ Department of Electrical Engineering, University of Texas at Dallas, Richardson, TX, USA.

Received: 30 January 2019 Accepted: 29 July 2019

Published online: 07 August 2019

\section{References}

1. Livingston G, Sommerlad A, Orgeta V, Costafreda SG, Huntley J, Ames D, et al. Dementia prevention, intervention, and care. Lancet. 2017;390(10113): 2673-734.

2. Lin FR, Metter EJ, O'Brien RJ, Resnick SM, Zonderman AB, Ferrucci L. Hearing loss and incident dementia. Arch Neurol. 2011;68(2):214-20.

3. Lin FR, Thorpe R, Gordonsalant S, Ferrucci L. Hearing loss prevalence and risk factors among older adults in the United States. J Gerontol. 2011;66(5): 582.

4. Criter RE, Honaker JA. Audiology patient fall statistics and risk factors compared to non-audiology patients. Int J Audiol. 2016;55(10):564-70.

5. Criter RE, Honaker JA. Fall risk screening protocol for older hearing clinic patients. Int J Audiol. 2017:56(10):1-8.

6. Brewster KK, Ciarleglio A, Brown PJ, Chen C, Kim HO, Roose SP, et al. Agerelated hearing loss and its association with depression in later life. Am J Geriatr Psychiatry. 2018;26(7):788-96.

7. Rutherford BR, Brewster K, Golub JS, Kim AH, Roose SP. Sensation and psychiatry: linking age-related hearing loss to late-life depression and cognitive decline. Am J Psychiatry. 2017. https://doi.org/10.1176/appi.ajp.201 7.17040423.

8. Mick P, Kawachi I, Lin FR. The association between hearing loss and social isolation in older adults. Otolaryngol Head Neck Surg. 2014;150(3):378-84.

9. Gopinath B, Schneider J, Mcmahon CM, Teber E, Leeder SR, Mitchell P. Severity of age-related hearing loss is associated with impaired activities of daily living. Age Ageing. 2012;41(2):195-200.

10. Hogan A, O'Loughlin K, Davis A, Kendig H. Hearing loss and paid employment: Australian population survey findings. Int J Audiol. 2009;48(3): 117-22.

11. Genther DJ, Frick KD, Chen D, Betz J, Lin FR. Association of hearing loss with hospitalization and burden of disease in older adults. JAMA. 2013;309(22): 2322-4.

12. Mathers C, Loncar D. Projections of global mortality and burden of disease from 2002 to 2030. PLoS Med. 2006:3(11):e442

13. Huddle MG, Goman AM, Kernizan FC, Foley DM, Price C, Frick KD, et al. The economic impact of adult hearing loss: a systematic review. JAMA Otolaryngol Head Neck Surg. 2017;143(10):1040-8. 
14. Foley DM, Frick KD, Lin FR. Association between hearing loss and healthcare expenditures in older adults. J Am Geriatr Soc. 2014;62(6):1188-9.

15. Economics BA. Listen hear! The economic impact and cost of hearing loss in Australia. Access Economics. 2006.

16. Grandori F, Lerch M, Tognola G, Paglialonga A, Smith P. Adult hearing screening: an undemanded need. Eur Geriatr Med. 2013:4(1):S4-5.

17. Goman AM, Lin FR. Prevalence of hearing loss by severity in the United States. Am J Public Health. 2016;106(10):e1.

18. Wade C, Lin FR. Prevalence of hearing aid use among older adults in the United States. Arch Intern Med. 2012;172(3):292-3.

19. Gates GA, Murphy MRees TS, Fraher A. Screening for handicapping hearing loss in the elderly. J Fam Pract. 2003:52(1):56-62.

20. Chou R, Dana T, Bougatsos C. Screening older adults for impaired visual acuity: a review of the evidence for the U.S. preventive services task force. Ann Intern Med. 2011;154(5):347.

21. Johnson CE, Danhauer JL, Koch LL, Celani KE, Lopez IP, Williams VA. Hearing and balance screening and referrals for Medicare patients: a National Survey of primary care physicians. J Am Acad Audiol. 2008;19(2):171-90.

22. Tomioka K, Ikeda H, Hanaie K, Morikawa M, Iwamoto J, Okamoto N, et al. The hearing handicap inventory for elderly-screening (HHIE-S) versus a single question: reliability, validity, and relations with quality of life measures in the elderly community, Japan. Qual Life Res. 2013;22(5):1151-9.

23. Yue W, Mo L, Li Y, Zheng Z, Yu Q. Analysing use of the Chinese HHIE-S for hearing screening of elderly in a northeastern industrial area of China. Int J Audiol. 2016:56(4):242-7.

24. Abughanem S, Handzel O, Ness L, Benartziblima M, Faitghelbendorf K, Himmelfarb M. Smartphone-based audiometric test for screening hearing loss in the elderly. Eur Arch Otorhinolaryngol. 2016;273(2):333-9.

25. Livshitz L, Ghanayim R, Kraus C, Farah R, Even-Tov E, Avraham Y, et al. Application-based hearing screening in the elderly population. Ann Oto Rhinol Laryngol. 2017;126(1):36-41.

26. Ramsey T, Svider PF, Folbe AJ. Health burden and socioeconomic disparities from hearing loss: a global perspective. Otol Neurotol. 2001;96(454):589-604.

27. Kim H, Loh WY. Classification trees with unbiased multiway splits. J Am Stat Assoc. 2001;96(454):589-604.

28. Fokkema M, Smits N, Kelderman H, Penninx BW. Connecting clinical and actuarial prediction with rule-based methods. Psychol Assess. 2015;27(2): 636-44

29. Strobl C, Malley J, Tutz G. An introduction to recursive partitioning: rationale, application, and characteristics of classification and regression trees, bagging, and random forests. Psychol Methods. 2009;14(4):323-48.

30. Stromberg KA, Agyemang AA, Graham KM, Walker WC, Sima AP, Marwitz JH, et al. Using decision tree methodology to predict employment after moderate to severe traumatic brain injury. J Head Trauma Rehabil. 2019; 34(3):E64-e74.

31. Sanchez Lopez R, Bianchi F, Fereczkowski M, Santurette S, Dau T. Datadriven approach for auditory profiling and characterization of individual hearing loss. Trends Hear. 2018;22:2331216518807400.

32. Qiu J, Yu C, Ariyaratne TV, Foteff C, Ke Z, Sun Y, et al. Cost-effectiveness of pediatric Cochlear implantation in rural China. Otol Neurotol. 2017;38(6):e75-84.

33. Hojjat H, Svider PF, Davoodian P, Hong RS, Folbe AJ, Eloy JA, et al. To image or not to image? A cost-effectiveness analysis of MRI for patients with asymmetric sensorineural hearing loss. Laryngoscope. 2017;127(4):939-44.

34. Burke MJ, Shenton RC, Taylor MJ. The economics of screening infants at risk of hearing impairment: an international analysis. Int J Pediatr Otorhinolaryngol. 2012;76(2):212-8.

35. Chiou ST, Lung HL, Chen LS, Yen AM, Fann JC, Chiu SY, et al. Economic evaluation of long-term impacts of universal newborn hearing screening. Int J Audiol. 2017:56(1):46-52

36. Leung J, Wang NY, Yeagle JD, Chinnici J, Bowditch S, Francis HW, et al. Predictive models for cochlear implantation in elderly candidates. Arch Otolaryngol Head Neck Surg. 2005;131(12):1049-54.

37. Zhao Y, Li J, Zhang M, Lu Y, Xie H, Tian Y, et al. Machine learning models for the hearing impairment prediction in workers exposed to complex industrial noise: a pilot study. Ear Hear. 2019;40(3):690-9.

38. Aliabadi M, Farhadian M, Darvishi E. Prediction of hearing loss among the noise-exposed workers in a steel factory using artificial intelligence approach. Int Arch Occup Environ Health. 2015;88(6):779-87.

39. Kamiński B, Jakubczyk M, Szufel P. A framework for sensitivity analysis of decision trees. CEJOR. 2018:26(1):135-59.
40. Louppe G. Understanding random forests: from theory to practice. 2014 Eprint Arxiv.

41. Breiman L, Friedman JH, Olshen RA, Stone CJ. Classification and regression trees. Monterey: Wadsworth \& Brooks/Cole Advanced Books \& Software; 1984.

42. Friedman $\mathrm{JH}$. A tree-structured approach to nonparametric multiple regression. Heidelberg: Springer Berlin; 1979. p. 5-22.

43. Quinlan JR. Induction of decision trees. Mach Learn. 1986;1(1):81-106.

44. Nafe R, Choritz H. Introduction of a neuronal network as a tool for diagnostic analysis and classification based on experimental pathologic data. Exp Toxicol Pathol. 1992:44(1):17-24.

45. Jiang L, Zhang H, Cai Z. A novel Bayes model: hidden naive Bayes. IEEE Trans Knowl Data Eng. 2009;21(10):1361-71.

46. Jensen FV. An introduction to Bayesian networks. London: UCL press; 1996; 210:1-178.

47. Breiman L. Random forests. Mach Learn. 2001;45(1):5-32.

48. Kamil RJ, Betz J, Powers BB, Pratt S, Kritchevsky S, Ayonayon HN, et al. Association of Hearing Impairment with incident frailty and falls in older adults. J Aging Health. 2015;28(4):644-60.

49. Gispen FE, Chen DS, Genther DJ, Lin FR. Association between hearing impairment and lower levels of physical activity in older adults. J Am Geriatr Soc. 2014:62(8):1427-33.

50. Genther DJ, Betz J, Pratt S, Kritchevsky SB, Martin KR, Harris TB, et al. Association of hearing impairment and mortality in older adults. J Gerontol A Biol Sci Med Sci. 2015;70(1):85-90.

51. Manrique-Huarte R, Calavia D, Huarte IA, Girón L, Manrique-Rodríguez M. Treatment for hearing loss among the elderly: auditory outcomes and impact on quality of life. Audiol Neurootol. 2016;21(Suppl 1):29-35.

52. Holte RC, editor. Exploiting the cost (in) sensitivity of decision tree splitting criteria. Seventeenth international conference on machine learning; 2000.

53. Shannon CE, Weaver W. The mathematical theory of communication. Bell Labs Tech J. 1950;3(9):31-2.

54. Raileanu LE, Stoffel K. Theoretical comparison between the Gini index and information gain criteria. Ann Math Artif Intell. 2004;41(1):77-93.

55. Williams CKI. Learning with kernels: support vector machines, regularization, optimization, and beyond. Publ Am Stat Assoc. 2003;98(462):489.

56. Belgiu $M$, Drăgut $L$. Random forest in remote sensing: a review of applications and future directions. ISPRS J Photogramm Remote Sens. 2016; 114:24-31.

57. Lai Z, Deng H. Medical image classification based on deep features extracted by deep model and statistic feature fusion with multilayer perceptron(). Comput Intell Neurosci. 2018;2018:2061516.

58. Pedregosa F, Varoquaux G, Gramfort A, Michel V, Thirion B, Grisel O, et al. Scikit-learn: machine learning in Python. J Mach Learn Res. 2013;12(10): 2825-30.

59. Ventry IM, Weinstein BE. The hearing handicap inventory for the elderly: a new tool. Ear Hear 1982;9(2):128-34.

60. Ciurlia-Guy E, Cashman M, Lewsen B. Identifying hearing loss and hearing handicap among chronic care elderly people. Gerontologist. 1993;33(5):644-9.

61. Maguire N, Prosser S, Boland R, Mcdonnell A. Screening for hearing loss in general practice using a questionnaire and the whisper test. Eur J Gen Pract. 1998:4(1):18-21.

62. Cruickshanks KJ, Wiley TL, Tweed TS, Klein BE, Klein R, ., Mares-Perlman JA, et al. Prevalence of hearing loss in older adults in Beaver Dam, Wisconsin. The epidemiology of hearing loss study. Am J Epidemiol 1998;148(9):879.

63. Bamini G, Elena R, Jin WJ, Julie S, Leeder SR, Paul M. Prevalence of agerelated hearing loss in older adults: Blue Mountains study. Arch Intern Med. 2009;169(4):415-6

64. Homans NC, Metselaar RM, Dingemanse JG, Mp VDS, Brocaar MP, Wieringa $\mathrm{MH}$, et al. Prevalence of age-related hearing loss, including sex differences, in older adults in a large cohort study. Laryngoscope. 2017;127(3):725-30.

65. Pittman AL, Stelmachowicz PG. Hearing loss in children and adults: audiometric configuration, asymmetry, and progression. Ear Hear. 2003;24(3): $198-205$.

\section{Publisher's Note}

Springer Nature remains neutral with regard to jurisdictional claims in published maps and institutional affiliations. 